Pontifícia Universidade Católica $_{\text {dita }}$

Lygia Alessandra Magalhães Magacho

\title{
Parque de Inovação de Serviços para as Pessoas Metodologias para o planejamento
}

Dissertação apresentada ao Programa de Pós-graduação em Administração de Empresas da PUC-Rio como requisito parcial para a obtenção do título de mestre em Administração de Empresas.

Orientadora: Profa. Maria Angela Campelo de Melo 
Lygia Alessandra Magalhães Magacho

\title{
Parque de Inovação de Serviços para as Pessoas: \\ Metodologias para o planejamento
}

Dissertação apresentada como requisito parcial para obtenção do grau de Mestre pelo Programa de Pósgraduação em Administração de Empresas da PUC-Rio. Aprovada pela Comissão Examinadora abaixo assinada.

\author{
Profa. Maria Angela Campelo de Melo \\ Orientadora \\ Departamento de Administração - PUC-Rio
}

Profa . Maria de Fátima Ludovico de Almeida

Departamento de Engenharia Industrial - PUC-Rio

Prof. Sérgio José Mecena da Silva Filho Universidade Federal Fluminense

Profa. Mônica Herz

Vice-Decana de Pós-Graduação do CCS-PUC-Rio

Rio de Janeiro, 15 de setembro de 2010 
Todos os direitos reservados. É proibida a reprodução total ou parcial do trabalho sem autorização da universidade, da autora e do orientador.

\section{Lygia Alessandra Magalhães Magacho}

Advogada, bacharel em Direito pela Faculdade da Cidade, com MBA em Administração de Empresas pelo IAG PUC- Rio. Trabalhou no Instituto Gênesis da PUC-Rio por 10 anos, atuando nos últimos 4 anos como Assessora da Direção, neste período acumulou experiência na área de direito e administração, com ênfase em temas como incubação de empresas, inovação, ecossistemas de inovação, desenvolvimento local, hélice tripla e alianças em rede. Atualmente é gerente de negócios do Grupo Parmê.

Ficha Catalográfica

Magacho, Lygia Alessandra Magalhães

Parque de inovação de serviços para as pessoas: metodologias para o planejamento / Lygia Alessandra Magalhães Magacho ; orientadora: Maria Angela Campelo de Melo. - 2010.

200 f. : il. (color.) ; $30 \mathrm{~cm}$

Dissertação (mestrado)-Pontifícia Universidade Católica do Rio de Janeiro, Departamento de Administração, 2010.

Inclui bibliografia

1. Administração - Teses. 2. Parques de inovação. 3. Planejamento adaptativo. 4. Sistemas de inovação. 5. Parque de inovação de serviços para as pessoas. 6. Rede iberoamericana de parques de inovação de serviços para as pessoas. I. Melo, Maria Angela Campelo de. II. Pontifícia Universidade Católica do Rio de Janeiro. Departamento de Administração. III. Título. 
A Deus, sempre e em primeiro Iugar.

Aos meus paise irmao. 


\section{Agradecimentos}

A meu pai e minha mãe pelas características e exemplos complementares de seres humanos e profissionais e por terem me apoiado no trilhar do meu próprio caminho. Ao meu irmão, por ter me ensinado que existe o outro e que não vivemos sozinhos, por isso a importância de saber dividir e aceitar. Aos meus avós que construíram a minha a história.

A minha segunda família que esteve ao meu lado em todos os momentos e conquistas, padrinhos Solange Brum Santos e Hebert Santos, irmãos de coração Daniel e Roberta Brum.

As amigas de infância, Julia Zardo e Taís Nasser que continuaram ao meu lado e se revelaram excelentes amigas de trabalho. Às amigas de Triade Gestão Empresarial, meu desafio empreendedor, Priscila Castro e Roberta Alves.

Ao meu eterno chefe, José Alberto Sampaio Aranha, por cada dia de aprendizado e de exemplos profissionais e pessoais, mas principalmente por me ensinar a comemorar cada passo para a conquista, e não, e tão somente, a própria.

Aos queridos professores Cesar e Helene Salim mentores de minha vida profissional e grandes amigos.

A querida Profa. Maria Angela Campelo de Melo incansável na tarefa de me orientar, tornando os dias de trabalho árduo em momentos agradáveis.

Aos amigos do mestrado que me acompanharam, dividiram conhecimentos, compartilharam desânimo e cansaço, mas que sempre me incentivaram, e que certamente ficarão: Marcos Caiado, Marcelo Presa e Mila Viana. 


\section{Resumo}

Magacho, Lygia Alessandra Magalhães; Melo, Maria Angela Campelo de. Parque de Inovação de Serviços para as Pessoas Metodologias para o planejamento. Rio de Janeiro, 2010. 200p. Dissertação de Mestrado Departamento de Administração, Pontifícia Universidade Católica do Rio de Janeiro.

Esta dissertação tem como objetivo identificar metodologias adequadas ao processo de planejamento do Parque de Inovação de Serviços para as Pessoas da Baixada Fluminense. Esse Parque faz parte de uma rede iberoamericana e visa provocar uma mudança social na região onde está inserido, melhorando a qualidade de vida e o bem estar social, por meio da transferência de conhecimento, tecnologia e inovação e da criação de empresas focadas no desenvolvimento de produtos e serviços que diminuam as desigualdades entre as classes sociais. Nesta dissertação foram utilizados o método de estudo de caso e a pesquisa-ação e analisadas teorias sobre planejamento de sistemas complexos e planejamento adaptativo, considerando as especificidades apresentadas pelos Parques Científicos e Tecnológicos e Parques de Inovação. O estudo aprofunda o entendimento sobre a problemática inerente ao planejamento dos parques, identifica os aspectos fundamentais a serem considerados durante o planejamento, caracteriza o parque como um sistema complexo, ativo e adaptativo e sugere metodologias mais adequadas ao seu planejamento.

\section{Palavras-chave}

Parques de inovação; planejamento adaptativo; sistemas de inovação; Parque de Inovação de Serviços para as Pessoas; Rede Iberoamericana de Parques de Inovação de Serviços para as Pessoas. 


\section{Abstract}

Magacho, Lygia Alessandra Magalhães; Melo, Maria Angela Campelo de (Advisor). Innovation Park for People Service - methodology for planning. Case Study of the Innovation Park for People Service. Rio de Janeiro, 2010. 200p. MSc. Dissertation - Departamento de Administração, Pontifícia Universidade Católica do Rio de Janeiro.

This dissertation aims at identifying appropriate methodologies for planning for the Innovation Park for People Service at Baixada Fluminense. This park, which is part of an Ibero-American network, was conceived to generate social changes in the region where it operates, improving the quality of life and social welfare, by transferring knowledge, technology and innovation and creating companies focused on the development of products and services to reduce social classes' disparities. The method of case study and action-research is adopted and theories about the planning of complex systems and adaptive planning are examined, considering the specificities of Science and Technology Parks and Innovation Parks. The study focuses on the understanding of park planning problems, identifies the key issues to be considered during this process, characterizes the park as an active adaptive complex system, and suggests methodologies for improving its planning process.

\section{Keywords}

Innovation Park, adaptive planning, innovation systems, Innovation Parks for People Service, Iberoamerican Network for Innovation Parks for People Service. 


\section{Sumário}

Lista de Figuras, Tabelas e Quadros

Lista de Siglas

1. Introdução 15

$\begin{array}{ll}\text { 1.1. Problema de Pesquisa } & 15\end{array}$

1.2. Objetivo 18

1.3. Delimitações do Estudo 19

1.4. Relevância do Estudo 20

1.5. Organização do Estudo 21

2. Ambientes de Inovação 22

2.1. Sistemas Nacionais de Inovação 22

2.2. Sistema Brasileiro de Inovação 23

2.3. Hélice Tríplice 27

3. Parques Científicos e Tecnológicos 31

3.1. Conceitos 31

3.2. Principais Parques Científicos e Tecnológicos 39

3.2.1. Estados Unidos 40

3.2.2. Europa 44

3.2.3. Ásia 51

3.2.4. América do Sul 52

3.3. Parques Científicos e Tecnológicos no Brasil 57

4. Planejamento de Sistemas Complexos 64

4.1. Sistemas Complexos 64

4.2. Problemática e Planejamento dos Parques Científicos e Tecnológicos e de Inovação 65

4.3. Processos de Planejamento Adaptativo e suas Metodologias 67

5. Método de Pesquisa 76

$\begin{array}{ll}\text { 5.1. Tipo de Pesquisa } & 76\end{array}$ 
$\begin{array}{ll}\text { 5.2. Pesquisa-ação } & 78\end{array}$

5.3. Desenvolvimento do Estudo 80

5.4. Limitações do Método 82

6. Estudo de Caso 84

6.1. A origem: a Rede de Parques de Inovação de Serviços para as Pessoas 84

6.2. O Modelo: Parque de Inovação de Serviços para as Pessoas 87

6.2.1. Fundamentos 89

6.3. A Região 92

6.4. PUC-Rio e o Instituto Gênesis 96

6.5. O Parque de Inovação de Serviços para as Pessoas da Baixada

$\begin{array}{ll}\text { Fluminense } & 97\end{array}$

6.6. Parque de Inovação da Baixada Fluminense: um Sistema Complexo 101

6.7. O Parque enquanto Sistema Ativo Adaptativo e as Metodologias Propostas 108

$\begin{array}{ll}\text { 7. Considerações Finais } & 115\end{array}$

$\begin{array}{ll}\text { Bibliografia } & 118\end{array}$

Anexo I - Documento Marco de Constituição da Rede Iberoamericana de Parques de Inovação de Serviços para as Pessoas.

Anexo II - Programa do Curso de Diretores realizado pela Rede Iberoamericana.

Anexo III - Apresentação do Parque de Inovação de Serviços para as Pessoas da Baixada Fluminense.

Anexo IV - Lista de Examinadores da Proposta de Parques Membros da Rede Iberoamericana. 


\section{Lista de Figuras, Tabelas e Quadros}

Figura 1- Modelo evolutivo da Hélice Tríplice 28

Figura 2: Evolução das Abordagens de Planejamento 69

Figura 3: Tipologias das Estratégias Contingenciais de Mudança 72

Figura 4: Integrantes da Rede Iberoamericana de Parques de Inovação de Serviços para as Pessoas

Figura 5: Modelo Geral de um Parque de Inovação de Serviços para as Pessoas

89

Figura 6: Mapa dos Municípios da Baixada Fluminense 96

Figura 7: Modelo do Parque de Inovação de Serviços para as Pessoas da Baixada

Gráfico 1- Crescimento e Geração de Parques Tecnológicos no Mundo

Gráfico 2: Crescimento dos Parques Tecnológicos no Mundo, 19502006

Gráfico 3: Parques Tecnológicos no Mundo com planos de expansão

Gráfico 4: Estágios dos Parques Tecnológicos no Brasil 57

Gráfico 5: Data de Início dos Parques Tecnológicos no Brasil 58

Gráfico 6: Estágios e Regiões dos Parques Tecnológicos no Brasil 58

Gráfico 7: Receitas, Exportação e Impostos dos Parques Tecnológicos no Brasil

Gráfico 8: Postos de Trabalho nos Parques Tecnológicos no Brasil

Gráfico 9: Número de Empresas por faturamento nos Parques Tecnológicos

Gráfico 10: Investimentos Realizados em Parques Tecnológicos no Brasil

Gráfico 11: Novos Investimentos nos Parques Tecnológicos no Brasil

60

Gráfico 12: Investimentos em Parques Tecnológicos no Brasil

Tabela 1: População da Baixada Fluminense

Tabela 2: Problemática dos Parques Científicos e Tecnológicos, dos Parques de Inovação de Serviços para as Pessoas e do PISP da Baixada Fluminense 


\section{Lista de Siglas}

ABDI - Agência Brasileira de Desenvolvimento Industrial

AECID - Agência Espanhola de Cooperação Internacional para o

Desenvolvimento

AGT - Agência de Gestão Tecnológica e Propriedade Intelectual

ANPROTEC - Associação Nacional de Entidades Promotoras de

Empreendimentos Inovadores

APSTI - Associazione dei Parchi Scientifici e Tecnologici Italiani

APTE - Associação de Parques Científicos e Tecnológicos da Espanha

ASPA - Asian Science Park Association

AURP - Association of University Research Parks

BBVA - Banco Bilbao Vizcaya Argentaria

BNDES - Banco Nacional de Desenvolvimento

C\&T - Ciência e Tecnologia

CENPES - Centro de Pesquisas e Desenvolvimento Leopoldo Américo Miguez

de Mello

CEPEL - Centro de Pesquisa em Energia Elétrica

CETEM - Centro de Tecnologia Mineral

CIDE - Centro de Informações e Dados do Rio de Janeiro

CNPq - Conselho Nacional de Desenvolvimento Científico e Tecnológico

COPPE - Instituto Alberto Luiz Coimbra de Pós-Graduação e Pesquisa de

Engenharia

DEI - Departamento de Engenharia Industrial

DNA - Deoxyribonucleic acid

Embraer - Empresa Brasileira de Aeronáutica S.A.

Embrapa - Empresa Brasileira de Pesquisa Agropecuária

ERBI - Europe's largest life science and healthcare cluster

FINEP - Financiadora de Estudos e Projetos

FUNTEC - Fundo Tecnológico

GTP - Gyeonggi Technopark

IASP - International Association of Science Parks

IBGE - Instituto Brasileiro de Geografia e Estatística

IBM - International Business Machines

IDH - Índice de Desenvolvimento Humano

IEN - Instituto de Engenharia Nuclear 
KRP - Kyoto Research Park Corp

MCT - Ministério de Ciência e Tecnologia

MDIC - Ministério de Desenvolvimento Industrial e Comércio

MIT - Massachusetts Institute of Technology

P\&D - Pesquisa e Desenvolvimento

$P+D+i-P e s q u i s a$, Desenvolvimento e Inovação

PE - Pernambuco

PERUINCUBA - Associação Peruana

Petrobras - Petróleo Brasileiro S.A.

PIB - Produto Interno Bruto

PISP - Parque de Inovação de Serviços para as Pessoas

PITCE - Política Industrial, Tecnológica e de Comércio Exterior

PqT -Parque Tecnológico

PTA - Parque Tecnológico de Andalucia

PTIC - Parque Tecnológico Industrial del Cerro

PTS - Parque Tecnológico Sartenejas

PTU-LUZ - Parque Tecnológico Universitario Del Zulia

PUC/RS - Pontifícia Universidade Católica do Rio Grande do Sul

PUC-Rio - Pontifícia Universidade Católica do Rio de Janeiro

RAHE - Programa de Capacitação de Recursos Humanos para Atividades

Estratégicas

RGE - Rio Grande Energia

RJ - Rio de Janeiro

RTP - Research Triangle Park

SBI - Sistema Brasileiro de Inovação

SC - Santa Catarina

SLI - Sistema Local de Inovação

SNI - Sistema Nacional de Inovação

TGV - Train à grande vitesse

TI - Tecnologia da Informação

TIC - Tecnologia da Informação e Comunicação

UKSPA - United Kingdom Science Park Association

UNESCO - United Nations Educational, Scientific and Cultural Organization

USB - Universidade Simón Bolívar USP _

Universidade de São Paulo WAINOVA -

World Alliance for Innovation 
ZIRST- Zone pour /'Innovation et /es Realisations Scientifiques et Technologiques 
